\title{
Internet und internationale Politik
}

Aus verschiedenen Gründen ist das Internet in den letzten Jahren den Brennpunkten politischer Aufmerksamkeit immer näher gerückt. Entscheidend dafür ist wohl, dass inzwischen die gesamte Wertschöpfungskette - von Produktion über Verteilung und Konsumption - global von einem stabilen und offenen Informationsnetz abhängig geworden ist. Darüber hinaus ist es die Tatsache, dass das Internet als Informations- und Kommunikationsplattform in der Lage zu sein scheint, global ideologische Positionen zu verbreiten und einen zunehmenden Einfluss auf die Meinungsbildung in den immer noch unterschiedlichen Gesellschaftsformen gewinnt. Der erste Aspekt kann möglicherweise zu einer einheitlichen politischen Strategie aller an der ökonomischen Stabilisierung der Welt Beteiligten für den Umgang mit entsprechenden InternetServices führen. Beim zweiten Aspekt ist ein einheitliches Vorgehen jedoch derzeit fraglich, insbesondere weil kulturelle Grundwerte - wie das Recht auf freie Meinungsäußerung, der Wert und die Reichweite der Privatsphäre, das Recht auf informationelle Selbstbestimmung und Weitere - global nicht einheitlich bewertet werden.

Vor diesem Hintergrund ist es bedeutungsvoll, dass sich ein global einflussreiche Gremium, die G8, im Summit ihres Treffens im französischen Deauville am 26. und 27. Mai 2011 erstmals auf gemeinsame Positionen zum Internet festgelegt hat. Um das Gewicht und die weltweite Durchsetzbarkeit dieser Positionen bewerten zu können, ist ein Blick auf die G8Interna sinnvoll.

G8 Mitglieder sind: Frankreich, die USA, Großbritannien, Russland, Deutschland, Japan, Italien und Kanada. Gegründet wurde das Gremium 1975 mit sechs Mitgliedern, 1976 wurde Kanada und 1998 Russland aufgenommen. Die Europäische Union ist seit 1977 assoziiertes Mitglied. G8 versteht sich als Repräsentant der Industrienationen. Ihre Mitglieder erbringen - nach eigener Darstellung - 65\% des weltweiten Bruttosozialprodukts und wickeln $2 / 3$ des internationalen Handels ab. Sie repräsentieren aller- dings nur 15\% der Weltbevölkerung. Diese Daten assoziieren, dass G8 Positionen im Weltmaßstab häufig als hegemonistisch interpretiert werden.

Im Summit ${ }^{1}$ des G8-Meetings 2011 finden sich 18 Thesen zum Internet. Einige wichtige Aussagen sind im Folgenden unkommentiert zusammengefasst:

Die Rolle des Internet wird hervorgehoben

- für die Bürger als Informations- und Bildungsressource und als Möglichkeit zur Förderung von Freiheit, Demokratie und Menschenrechten;

- für die Unternehmen als unersetzliche Plattform für die Wirtschaftstätigkeit und als Treiber von Innovation und Effizienz für Wachstum und Entwicklung;

- für die Regierungen als Plattform für eine effektive Verwaltung und Bürgernähe sowie für die Respektierung und Unterstützung von Menschenrechten.

Als grundlegende Voraussetzungen für den Erfolg und die weitere Entwicklung des Internet werden Offenheit, Transparenz und Freiheit in Verbindung mit Nichtdiskriminierung und fairem Wettbewerb genannt.

- Diese Prinzipien müssen in Verbindung mit gesetzlichen Vorgaben, respektierten Menschenrechten und dem Schutz von Intellektuellem Eigentum implementiert werden. Dabei sollen gleichzeitig angemessene Relationen zwischen Freiheit und Sicherheit, Transparenz und Vertraulichkeit sowie der Ausübung von individuellen Rechten und Verantwortung erreicht werden.

- Die Meinungsfreiheit in all ihren Ausprägungen muss geschützt werden, Zensur von Inhalten und Zugangsbeschränkungen sind nicht akzeptabel.

- Für Internet-basierte Services sind Interoperabilität und Konvergenz durch öffentlichen Regelwerke zu fordern, ebenso der Schutz von personenbezogenen Daten, die Netzneutralität, der grenzüberschreitende Datenaustausch, die

1 http://www.g20-g8.com/g8-g20/g8/english/ live/news/renewed-commitment-for-freedom-anddemocracy.1314.html
Netzsicherheit und das Intellektuelle Eigentum.

- Ein wirkungsvoller Schutz von personenbezogenen Daten und der Privatsphäre bleibt die Grundlage für das Nutzervertrauen.

- Die Sicherheit der Netze und der Dienste im Internet kann nur durch alle Beteiligten gemeinsam erreicht werden Deshalb ist die Koordination zwischen Regierungen und internationalen Organisationen, der Privatwirtschaft und der Zivilgesellschaft notwendig, um das Netz vor Missbrauch durch Terroristen und kriminelle Organisationen zu schützen. Hierbei soll die Roma-Lyon-Group der G8 ${ }^{2}$ eine bedeutende Rolle spielen.

- Alle Beteiligten werden zum Schutz der Kinder vor schädlichen Inhalten aufgerufen. Das Ziel ist eine Internet-Umgebung für Kinder, die sich auf Ausbildung und auch Kontrolle durch die Eltern stützt.

G8 will alle kommenden Möglichkeiten nutzen, um die internationale Kooperation im Sinne der Thesen des G8-Meetings $2011 \mathrm{zu}$ intensivieren. Wichtige Veranstaltungen im Jahr 2011 sind:

Das OECD High Level Meeting „The Internet Economy: Generating Innovation and Growth"im Juni in Paris;

das Internet Governance Forum im September in Nairobi;

die International Cyber Conference im November in London;

die Conference on Copyright im November in Avignon.

Es ist zu erwarten, dass der Impuls der G8 in Europa und international lebhaft diskutiert werden wird. Für eine Umsetzbarkeit der Thesen wird entscheidend sein, wie sich die großen Schwellenländer positionieren.

Auf jeden Fall scheint es notwendig, die aktuellen Konzepte für die Entwicklung des Datenschutzrecht in die Findung von globalen Regeln für das Internet einzubringen.

2 http://www.auswaertiges-amt.de/DE/ Aussenpolitik/GlobaleFragen/G8/G8-Lyon-Gruppe_ node.html 